\title{
Entropy of mitochondrial DNA circulating in blood is associated with hepatocellular carcinoma
}

David S. Campo ${ }^{1 *}$, Vishal Nayak ${ }^{2,3}$, Ganesh Srinivasamoorthy ${ }^{2,3}$ and Yury Khudyakov ${ }^{1}$

From 7th IEEE International Conference on Computational Advances in Bio and Medical Sciences (ICCABS 2017)

Orlando, FL, USA. 19-21 October 2017

\begin{abstract}
Background: Ultra-Deep Sequencing (UDS) enabled identification of specific changes in human genome occurring in malignant tumors, with current approaches calling for the detection of specific mutations associated with certain cancers. However, such associations are frequently idiosyncratic and cannot be generalized for diagnostics. Mitochondrial DNA (mtDNA) has been shown to be functionally associated with several cancer types. Here, we study the association of intra-host mtDNA diversity with Hepatocellular Carcinoma (HCC).

Results: UDS mtDNA exome data from blood of patients with HCC $(n=293)$ and non-cancer controls $(N C, n=391)$ were used to: (i) measure the genetic heterogeneity of nucleotide sites from the entire population of intra-host mtDNA variants rather than to detect specific mutations, and (ii) apply machine learning algorithms to develop a classifier for HCC detection. Average total entropy of HCC mtDNA is 1.24-times lower than of NC mtDNA ( $p=2.84 \mathrm{E}-$ 47). Among all polymorphic sites, $2.09 \%$ had a significantly different mean entropy between HCC and NC, with 0. $32 \%$ of the HCC mtDNA sites having greater $(p<0.05)$ and $1.77 \%$ of the sites having lower mean entropy $(p<0.05)$ as compared to NC. The entropy profile of each sample was used to further explore the association between mtDNA heterogeneity and HCC by means of a Random Forest (RF) classifier The RF-classifier separated 232 HCC and 232 NC patients with accuracy of up to $99.78 \%$ and average accuracy of $92.23 \%$ in the 10-fold cross-validation. The classifier accurately separated $93.08 \%$ of HCC $(n=61)$ and NC $(n=159)$ patients in a validation dataset that was not used for the RF parameter optimization.

Conclusions: Polymorphic sites contributing most to the mtDNA association with HCC are scattered along the mitochondrial genome, affecting all mitochondrial genes. The findings suggest that application of heterogeneity profiles of intra-host mtDNA variants from blood may help overcome barriers associated with the complex association of specific mutations with cancer, enabling the development of accurate, rapid, inexpensive and minimally invasive diagnostic detection of cancer.
\end{abstract}

Keywords: Mitochondria, Liquid biopsy, Machine learning, Entropy

\footnotetext{
* Correspondence: fyv6@cdc.gov

'Division of Viral Hepatitis, Centers for Disease Control and Prevention,

Atlanta, GA, USA

Full list of author information is available at the end of the article
}

(c) The Author(s). 2019 Open Access This article is distributed under the terms of the Creative Commons Attribution 4.0 International License (http://creativecommons.org/licenses/by/4.0/), which permits unrestricted use, distribution, and reproduction in any medium, provided you give appropriate credit to the original author(s) and the source, provide a link to the Creative Commons license, and indicate if changes were made. The Creative Commons Public Domain Dedication waiver (http://creativecommons.org/publicdomain/zero/1.0/) applies to the data made available in this article, unless otherwise stated. 


\section{Background}

Cancer is the leading cause of morbidity and mortality worldwide, with the estimated 14 million new cases and 8.2 million cancer-related deaths in 2012, and this number is predicted to rise by $\sim 70 \%$ over the next two decades [1]. Successful clinical management of cancer patients is largely contingent on early tumor detection and accurate assessment of treatment efficacy [2]. Currently, the standard diagnostic procedure for cancer is histological analysis of tissue biopsy. However, biopsies have several disadvantages, as they are invasive, costly and time-consuming. Only highly trained pathologists can perform histological detection and characterization of cancer from the sampled tissue. In addition, although generally safe, biopsies may cause complications such as bleeding, infection and accidental injury to adjacent structures [2].

Further improvement of cancer patient care greatly depends on development of accurate, minimally invasive, inexpensive and rapid diagnostic techniques. Recent progress in the identification of cancer biomarkers opened a new field of cancer diagnostics (see [2] for a review). The rapid, cheap and non-invasive nature of the "liquid biopsy" has the potential to bring fundamental change to cancer care by allowing for a repeat sampling and testing of blood for the early disease detection and effective monitoring of treatment responses [3].

Tumors shed nucleic acids into blood, a phenomenon that was exploited since the early discovery of cancer-related DNA mutations [4-7]. Screening of the whole human genome, the exome or mitochondrial DNA allows for the detection of mutant DNA species associated with different malignant tumors (see [3] for a review). Detection of tumor DNA circulating in blood provides a direct measure of cancer rather than an indirect assessment of the effects of cancer. However, low concentration of the tumor DNA in blood hampers its use in diagnostics. Recently, ultra-deep sequencing (UDS) has been applied to the efficient detection of the tumor DNA [8], thus significantly facilitating early cancer detection in asymptomatic individuals. Such mutant DNA species can be detected even at a very low concentration in blood of patients [9]. However, the complex and variable genetic nature of cancer in each patient often hinders the identification of mutations suitable for cancer diagnostics (see [3] for a review).

Here, we show that heterogeneity profiles of the intra-host mtDNA population are strongly associated with Hepatocellular Carcinoma (HCC). The small size of mtDNA is especially suitable for the accurate assessment of such profiles, application of which to the HCC detection overcomes the often-idiosyncratic association of specific mutations to cancer. The findings in this study suggest that genetic diversity of intra-host mtDNA in blood may serve as a generalizable marker for the accurate, rapid, inexpensive and minimally invasive diagnostic detection of cancer.

\section{Methods \\ Datasets}

The dataset was obtained from The Cancer Genome Atlas (TCGA) Research Network [10] and tested under the TCGA approved project \#9811. TCGA generated the Illumina exome data from 11,079 patients and 34 different cancer types, including 376 patients with Hepatocellular Carcinoma (HCC). For detailed information on the clinical definition of HCC please refer to The Cancer Genome Atlas (TCGA) Research Network [10]. Figure 1 shows demographic characteristics of the HCC samples. For non-cancer controls (NC), data were obtained from the 1000 Genomes project [11]. This project holds UDS data from 2504 individuals of 26 human populations. From these, 293 samples were selected that satisfied the following criteria: (i) unrelated to each other; (ii) collected from same geographic regions as the HCC samples; (iii) same technology (Illumina) and same exome library preparation as HCC (Nimblegen); (iv) mtDNA genome coverage $>95 \%$, and $(\mathrm{v})$ overall match to $\mathrm{HCC}$ samples by gender and mtDNA lineages.

\section{Pre-processing}

Figure 2 shows the bioinformatics pipeline implemented here for pre-processing of sequence files. The input is an exome sequence file in the fastq format. The output is a standardized mtDNA entropy profile (SMEP). The pipeline is implemented in Python and optimized to run on a Linux cluster, taking in average of $30 \mathrm{~min}$ per set of 16 samples. The pipeline includes the following steps:

1. Reads were mapped to the mtDNA reference [12] using recommendations and parameters implemented in MTOOLBOX [13, 14]. Reads mapped to mtDNA were retained for further analyses.

2. Reads were further mapped to nuclear human genome to remove NUMTs (nuclear mitochondrial DNA segments) following the recommendations and parameters described in [13, 14].

3. PCR duplicates were removed using Picard MarkDuplicates (http://broadinstitute.github.io/ picard/)

4. Quality trimming was performed using FAQCS [15].

5. A read count profile was created using BAM-read Count (https://github.com/genome/bamreadcount).

6. Low frequency variants were separated from Illumina sequence errors following the procedure 
A

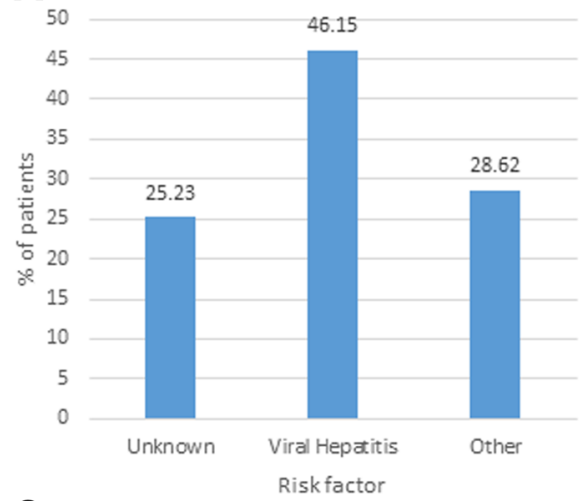

C

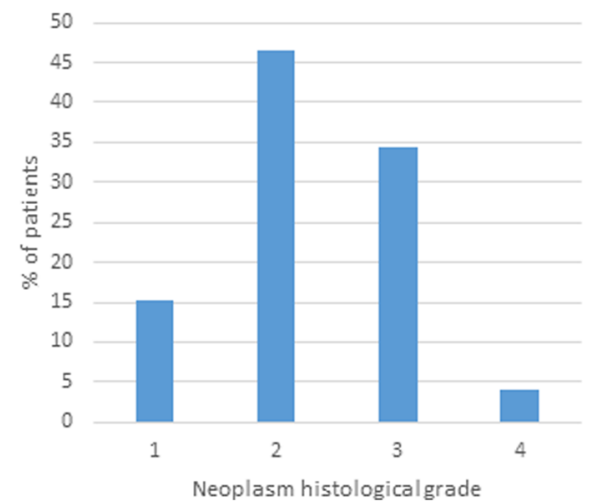

B
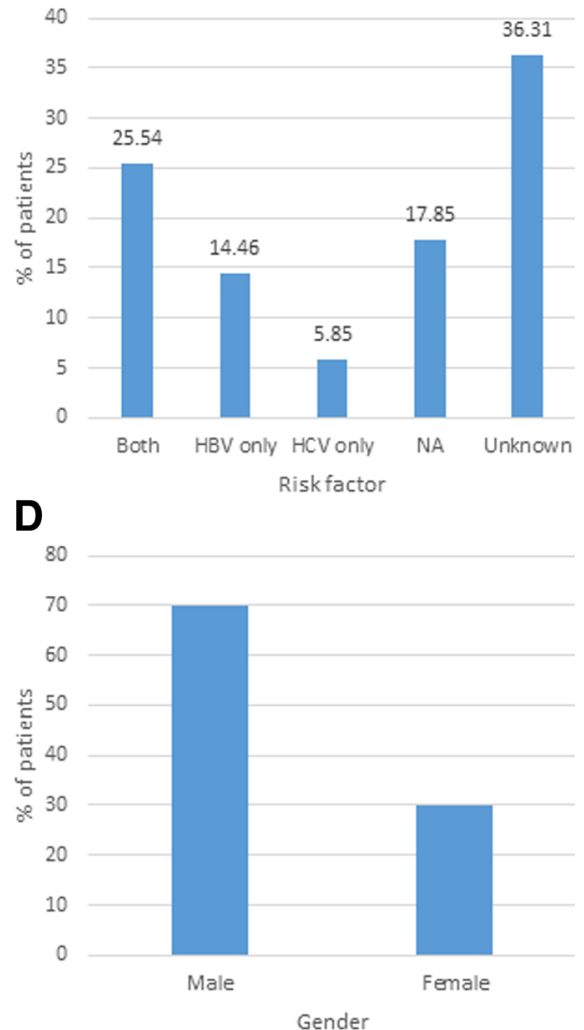

Fig. 1 Demographic characteristics of the cancer samples. a Risk factors; b Detail of Viral Hepatitis risk factors; c Neoplasm histological grade; d Gender

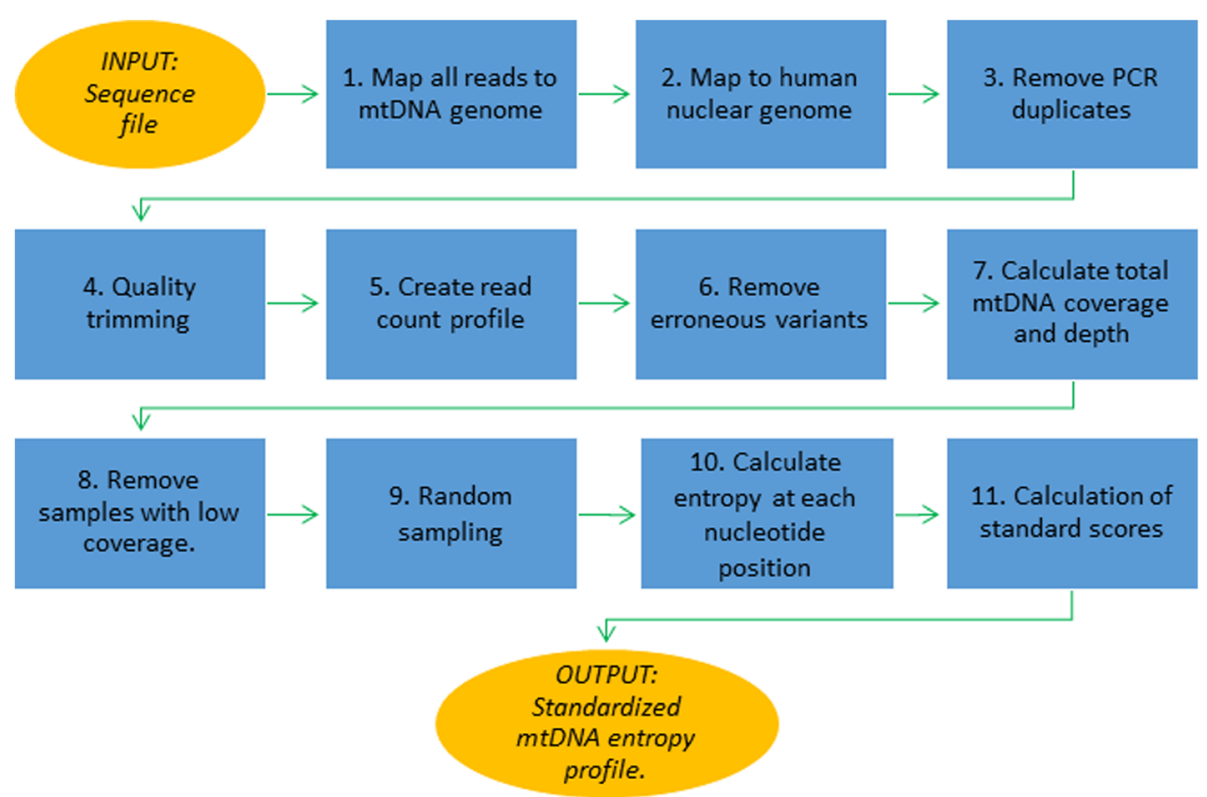

Fig. 2 Outline of the pre-processing of sequence files 
described in [16]. A variant is removed if the probability that it is an error was $>0.00001$.

7. The total mtDNA coverage and the depth at each position were caHCCulated.

8. Samples with a total coverage of $<95 \%$ of $\mathrm{mtDNA}$ are removed.

9. To reduce differences in genetic heterogeneity among files that were solely due to sampling depth, 100 random samples of 50 reads were taken at each mtDNA position. The target number of reads was chosen as this was the average depth found in the HCC dataset $(n=49.6)$.

10. Genetic heterogeneity at each nucleotide site for each of the 100 random samples was caHCCulated as an average of Shannon entropy [17] over all random samples. The Shannon entropy $H$ of a nucleotide site $j$ with $n$ different variants is given by:

$$
H_{j}=-\sum_{i=1}^{n} x_{i} \log _{b} x_{i}
$$

Where $x_{i}$ is the fraction of reads covering that position that show variant ${ }_{\mathrm{i}}$ and $b$ is the base of the logarithm (in this case, $b=2$ ).

11. Finally, to make the profiles more comparable and, thus, to increase the generalization power of the test, we transformed each heterogeneity profile into a set of Z-scores, the signed number of standard deviations by which each observation is above or below the mean of the sample. We found that this standardization greatly improved the accuracy of the classifier.

\section{Comparison of mtDNA from liver and blood of HCC patients}

The samples available from HCC patients included tumor $(n=358)$, normal liver $(n=85)$ and blood $(n=$ 293). We performed a detailed comparison of the mtDNA variants showed in the liver and blood of the same HCC patients, in terms of average number of reads, average depth of mtDNA sequencing, total mtDNA entropy, percentage of the mtDNA genome covered, percentage of all reads that map to mtDNA, and number of polymorphic positions. The purpose of this comparison is to show the extent of mtDNA changes observed in tumors and (ii) the degree of homogeneity of these mutations among HCC patients.

\section{Machine learning}

After obtaining SMEP for each sample, we studied the association between SMEP and $\mathrm{HCC} / \mathrm{NC}$ using the following steps:
1. We measured how the association level of each of the 16,569 nucleotide sites with the HCC/NC grouping my means of Iterative Relief $[18,19]$. Machine learning was repeated with different percentages of the 16,569 mtDNA sites (top 1\%, 5, $10 \ldots, 95$ and $100 \%$ ), with the top $1 \%$ showing the best results ( $1 \%=166$ sites $)$.

2. Supervised machine learning was performed using the Random Forest (RF) technique [20] as implemented in Sci-kit [21]. Although other methods, such as Nearest Neighbors, Nearest centroid, Support Vector Machine, Logistic regression, Gaussian Naïve Bayes, Decision trees and a Perceptron, were also tested, RF identified best genetic associations to the $\mathrm{HCC}$ and $\mathrm{NC}$ groups.

3. A grid search of the best combination of parameters was performed. The performance of each combination of parameters was measured using the 10 -fold cross-validation $(10 x C V)$. The final parameters of the classifier were the following: Number of trees: 101; Maximum tree depth: 4; Minimum number of instances to perform a split: 19. Splitting criterion: entropy; minimum number of instances in a leaf: 1; class weight: balanced.

4. Classifier with the highest $10 x C V$ accuracy was used to test a validation dataset that was not used for the parameter optimization.

In addition, we also tested the most heterogeneous mitochondrial genomic regions, HVS1 (positions 15,977-16,391 bp), which has been extensively used in many genetic studies. Reads covering this region were extracted from the exome data and used to generate the RF classifier using the same procedure described above.

\section{Results}

\section{mtDNA from liver and blood of HCC patients}

Figure 3 shows comparison among the samples' average number of reads, average depth of mtDNA sequencing, total mtDNA entropy, percentage of the mtDNA genome covered, percentage of all reads that map to mtDNA, and number of polymorphic positions. Pairwise comparison among 3 tissues in each HCC patient showed that, with the exception of the number of reads, the above parameters are significantly higher in normal liver (paired t-test; $p<0.05$ ), while the lowest values were detected in blood (Table 1), indicating a lower representation of mtDNA in blood as compared to liver and reduction in mtDNA in tumor as compared to normal liver.

Consensus sequences of mtDNA were generated for each tissue in each of the samples. On average, the consensus sequences of mtDNA found in tumors and blood of same patient differ at 0.92 sites, being identical in 

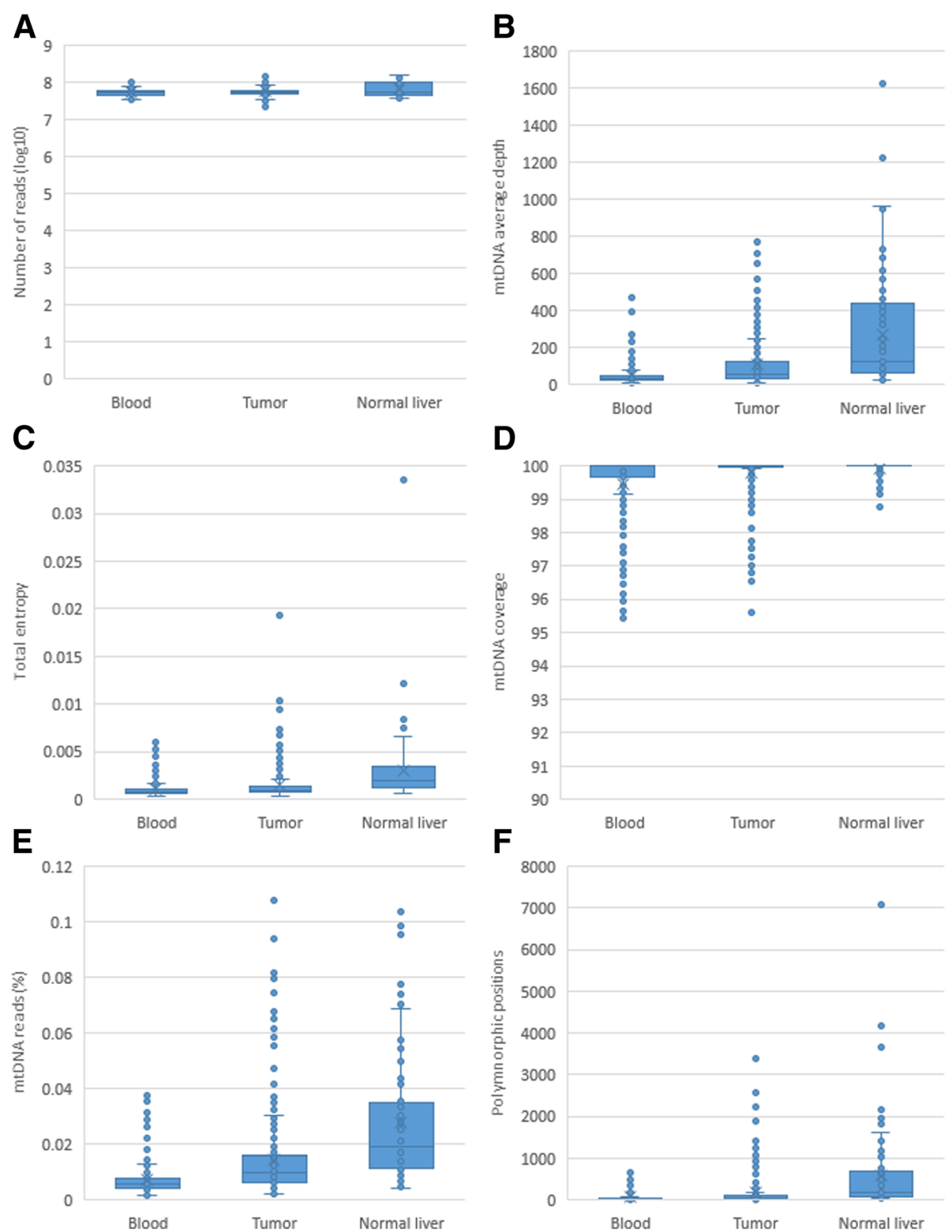

Fig. 3 Comparison between tissues of cancer patients. a Number of reads, all pairwise comparisons have a $p$ value; $\mathbf{b}$ mtDNA average depth; $\mathbf{c}$ mtDNA total entropy; $\mathbf{d}$ Percentage of the mtDNA genome covered; e Percentage of all reads that map to the mtDNA genome; $\mathbf{f}$ Number of polymorphic sites

$42.23 \%$ of the patients. Consensus sequences from tumor and normal tissue differ at 1.17 sites, being identical in only $37.04 \%$ of the individuals. Consensus sequences from blood and normal liver tissue of the same patient were much more similar, with an average difference at 0.16 sites and consensus sequences being identical in $84 \%$ of patients.

Pairwise comparison of UDS data from the three tissue samples identified 492 sites, entropy of which was significantly different between tumor and normal liver (paired t-test; $p<0.05$ ). However, only 38 of the sites differed between tumor and blood (paired t-test; $p<0.05$ ), while blood and tumor mtDNA differ at 319 sites $(p<$ $0.05)$. Despite significant similarity of consensus sequences, entropy of 468 sites differed in mtDNA from blood and normal liver $(p<0.05)$, indicating differences in intra-host mtDNA heterogeneity between these two tissues.

The consensus sequences from tumor and blood differed at 169 sites ("tumor-specific" sites) scattered across the entire genome (Fig. 4c). Mutations at these sites ("tumor-specific" mutations) were, however, present at low frequency in the blood of $7.03 \%$ of patients and in $18.95 \%$ of patients with a normal liver. Most of the 
Table 1 Comparison between tissues of HCC patients. Ratio of the averages and $p$ value of the paired samples t-test

\begin{tabular}{|c|c|c|c|c|c|c|}
\hline & $\begin{array}{l}\text { Blood vs Normal } \\
\text { liver }\end{array}$ & $p$ value & $\begin{array}{l}\text { Blood vs } \\
\text { Tumor }\end{array}$ & $p$ value & $\begin{array}{l}\text { Normal liver vs } \\
\text { Tumor }\end{array}$ & $p$ value \\
\hline Number of patients & 25 & N/A & 277 & N/A & 81 & N/A \\
\hline Number of reads $(\log 10)$ & 1.02 & $\begin{array}{l}8.78 \mathrm{E}- \\
01\end{array}$ & 0.99 & $\begin{array}{l}7.82 \mathrm{E}- \\
01\end{array}$ & 1.02 & $\begin{array}{l}7.82 \mathrm{E}- \\
01\end{array}$ \\
\hline mtDNA average depth & 0.14 & $\begin{array}{l}9.96 \mathrm{E}- \\
05\end{array}$ & 0.49 & $\begin{array}{l}8.30 \mathrm{E}- \\
12\end{array}$ & 1.86 & $\begin{array}{l}6.68 \mathrm{E}- \\
04\end{array}$ \\
\hline mtDNA total entropy & 0.49 & $\begin{array}{l}1.40 \mathrm{E}- \\
03\end{array}$ & 0.75 & $\begin{array}{l}2.22 \mathrm{E}- \\
03\end{array}$ & 1.26 & $\begin{array}{l}2.44 \mathrm{E}- \\
01\end{array}$ \\
\hline Percentage of the mtDNA genome covered & 0.98 & $\begin{array}{l}1.06 \mathrm{E}- \\
06\end{array}$ & 1.00 & $\begin{array}{l}1.95 \mathrm{E}- \\
06\end{array}$ & 1.00 & $\begin{array}{l}4.87 \mathrm{E}- \\
04\end{array}$ \\
\hline $\begin{array}{l}\text { Percentage of all reads that map to the mtDNA } \\
\text { genome }\end{array}$ & 0.19 & $\begin{array}{l}1.45 \mathrm{E}- \\
06\end{array}$ & 0.50 & $\begin{array}{l}2.02 \mathrm{E}- \\
12\end{array}$ & 1.72 & $\begin{array}{l}3.33 \mathrm{E}- \\
04\end{array}$ \\
\hline Number of polymorphic sites. & 0.18 & $\begin{array}{l}3.17 \mathrm{E}- \\
04\end{array}$ & 0.50 & $\begin{array}{l}8.29 \mathrm{E}- \\
04\end{array}$ & 2.09 & $\begin{array}{l}2.52 \mathrm{E}- \\
03\end{array}$ \\
\hline Number of different sites $(p<0.05)$ & 468 & N/A & 319 & N/A & 492 & N/A \\
\hline
\end{tabular}
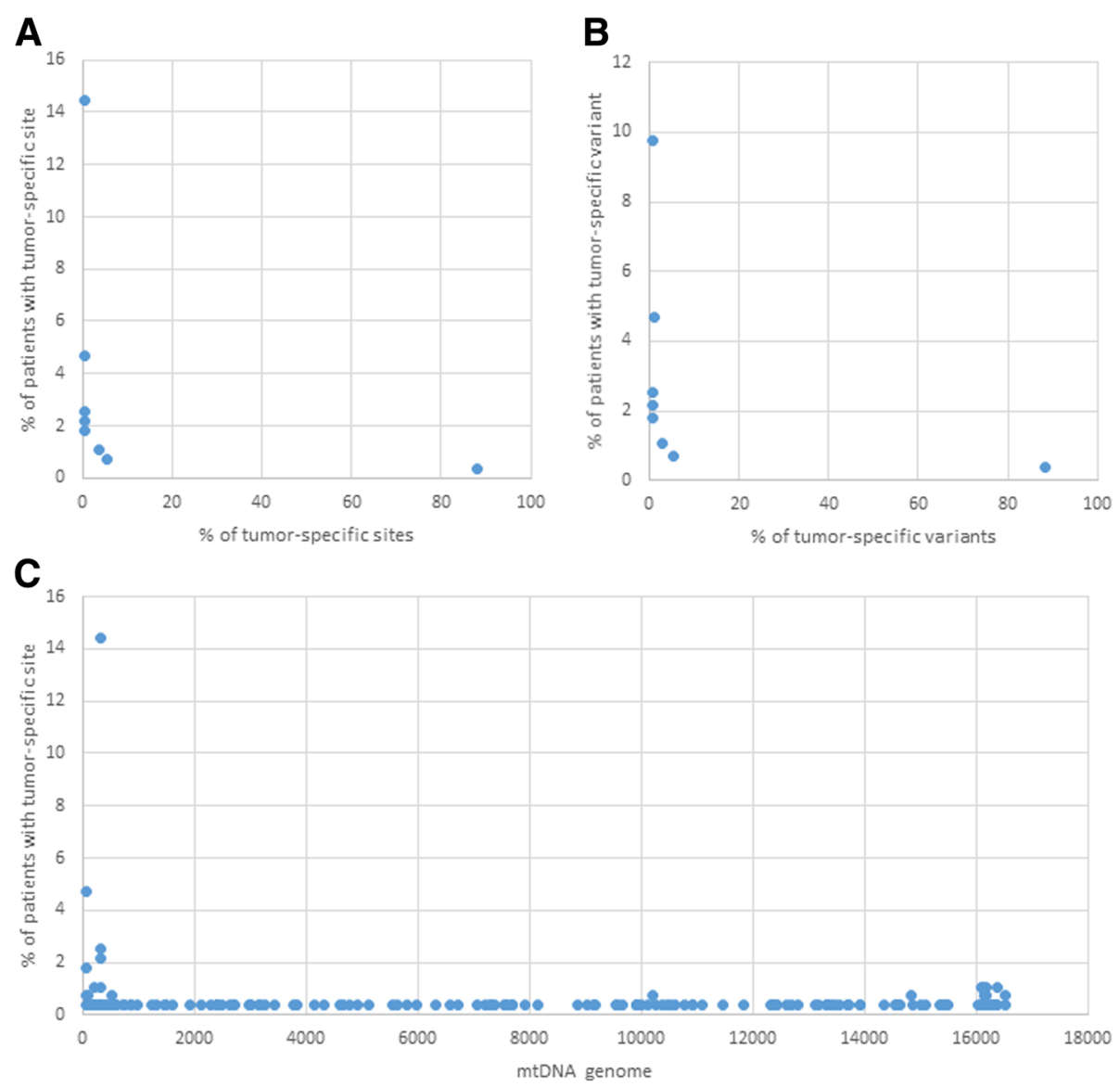

Fig. 4 Tumor-specific sites and variants in different HCC patients. a Percentage of tumor-specific sites that are present in several HCC patients; $\mathbf{b}$ Percentage of tumor-specific variants that are present in several HCC patients; c Distribution of tumor-specific sites along the genome 
tumor-specific mutations (88.16\%) were found only once in other HCC patients. Only one tumor-specific mutation at site 310 was present in $14.44 \%$ of the HCC patients (Fig. 4a). Both observations indicated a low association of these mutations with HCC.

\section{mtDNA in $\mathrm{HCC}$ and NC patients' blood}

Considering that mtDNA was tested in blood from all cases studied here, analyses on genetic differences in mtDNA between HCC and NC were focused on data from blood. The number of available samples, gender and mtDNA lineages between the HCC and NC groups were equalized to ensure statistical significance of observations on differences between these two groups. The two groups showed small but statistically significant differences in average entropy of mtDNA, percentage of exome reads mapped to mtDNA, percentage of all reads mapped to mtDNA, and number of polymorphic sites (Table 2).

When compared with NC, HCC have 1.24-times lower average total entropy $(p=2.84 \mathrm{E}-47)$ and 3.6-times lower percentage of all reads mapped to mtDNA $(p=$ 8.23E-19) (Table 2 and Fig. 5). Among all mtDNA polymorphic sites, $2.09 \%$ showed a significantly different mean entropy between HCC and NC. These selected sites were evenly distributed across the entire mtDNA. Only $0.32 \%$ of the sites had a higher mean entropy $(p<$ $0.05)$ but $1.77 \%$ had a lower mean entropy in $\operatorname{HCC}(p<$ $0.05)$. Thus, certain polymorphic sites scattered along mtDNA differed in the degree of diversity between HCC and NC patients, indicating their potential application as markers of HCC.

\section{Genetic association with HCC}

The top $1 \%$ of the mtDNA 16,569 nucleotide sites $(n=$ 166) with the highest Iterative Relief scores were used for the classifier optimization (Fig. 6a). These sites were not clustered in any gene but spread over mtDNA. The samples were separated into two groups, the first was used for the classifier optimization in 10-fold Cross-Validation $(10 \mathrm{xCV})$, and the second, which was not used for the optimization, was used for the final classifier testing. Figure $6 \mathrm{~b}$ shows the number of samples in each set. Using the first set, the RF-based classifier showed accuracy of up to $99.78 \%$ and an average accuracy in $10 x C V$ of $92.23 \%$. Finally, the RF classifier yielded an accuracy of $93.08 \%$ on the test dataset (Fig. 6c). All these data indicate that the mtDNA heterogeneity is strongly associated with $\mathrm{HCC}$ and $\mathrm{NC}$.

Among the top 1\% HCC-specific sites $(n=166)$ selected by ReliefF, only $11(6.6 \%)$ are shared with the "tumor-specific" sites $(n=169)$ selected using consensus sequences. Thus, although both are scattered across the entire mtDNA, individual sites from both groups are very different.

\section{HVS1 association with HCC}

Although polymorphic sites of significance found here are distributed along the entire mtDNA, we tested the performance of only the most heterogeneous mitochondrial genomic region, HVS1. The average $10 \mathrm{xCV}$ accuracy was $83.22 \%$, indicating that, although at the reduced rate, the distribution of sites' entropy in this region alone is strongly associated with HCC. However, it should be noted that increase in the coverage depth might help to identify more polymorphic sites in this region, thus potentially improving accuracy of classification. Application of UDS to a small genomic region could offer a greater control over sequencing depth, which is important for accurate assessment of genetic heterogeneity, especially in mtDNA extracted from blood where its concentration is low.

\section{Assessment of the neoplasm histologic grade}

In the TCGA HCC dataset, 292 samples had available information regarding the neoplasm histologic grade: 41 samples were classified as stage 1 cancer, 134 samples were stage 2, 104 samples were stage 3 and 13 samples were stage 4 (Fig. 2c). In $10 x C V$, the RF Regression yielded an average absolute error of 0.61 , which is only $2 \mathrm{X}$ better than the average absolute error of a random assignment (1.249), showing only a moderate association of SMEPs with the grades. Implementation of binary classification schemes instead of regression (e.g. Stage 1 vs all others) didn't improve classification accuracy.

\section{Discussion}

Analyses conducted in this study indicate that heterogeneity profiles of the intra-host mtDNA variants from

Table 2 Comparison between HCC and NC samples. Ratio of the averages and $p$ value of the paired samples t-test

\begin{tabular}{|c|c|c|c|c|}
\hline & $\mathrm{HCC}$ & NC & Ratio & $p$ value \\
\hline Number of reads $(\log 10)$ & 7.7429 & 7.4892 & 0.9672 & 2.62E-15 \\
\hline mtDNA average depth & 49.6176 & 120.0060 & 2.4186 & $1.73 \mathrm{E}-12$ \\
\hline mtDNA total entropy & 0.0011 & 0.0014 & 1.2798 & $1.96 \mathrm{E}-05$ \\
\hline Percentage of the mtDNA genome covered & 99.4562 & 99.6885 & 1.0023 & 0.004217 \\
\hline Percentage of all reads that map to the mtDNA genome & 0.0073 & 0.0388 & 5.3349 & $2.78 \mathrm{E}-30$ \\
\hline Number of polymorphic sites. & 68.4334 & 129.8362 & 1.8973 & $5.86 \mathrm{E}-06$ \\
\hline
\end{tabular}



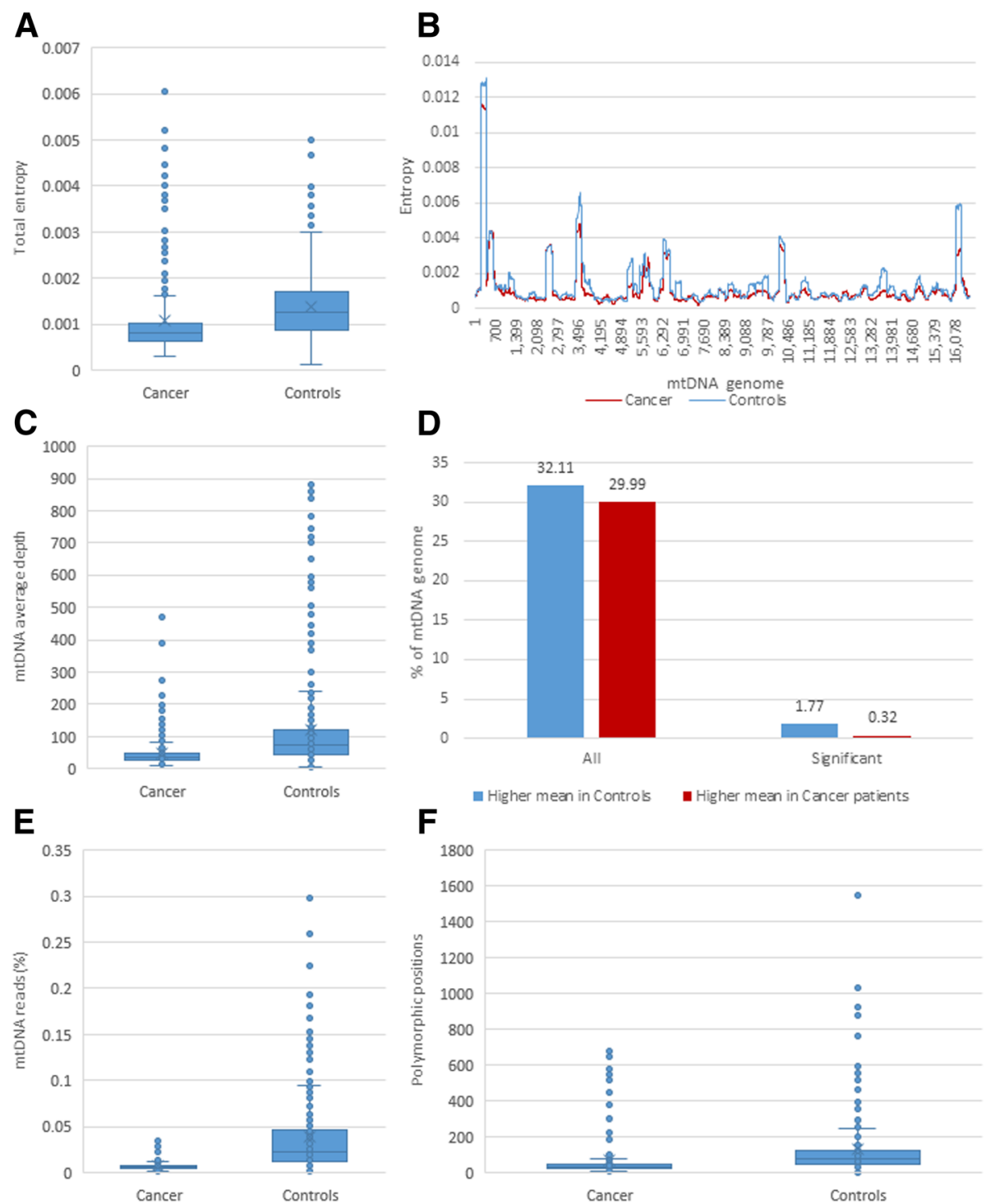

Fig. 5 Differences between HCC and NC samples. a Average entropy; b Average entropy over the mtDNA genome. Sliding moving window = $201 \mathrm{bp}$, step $=1$; $\mathbf{c}$ Percentage of all exome reads that map to the mtDNA genome; $\mathbf{d}$ Percentage of mtDNA sites with high average entropy; $\mathbf{e}$ Percentage of all reads that map to the mtDNA genome; $\mathbf{f}$ Number of polymorphic sites

blood are strongly associated with HCC. Although cancer detection is usually focused on genetic analysis of nuclear DNA [2], mtDNA has been shown to be functionally associated with several cancer types [22]. Owing to its clonal nature, high copy number and high mutation rate [23], mtDNA has many practical advantages over nuclear DNA in application to cancer detection. Mitochondria supply energy for all metabolic processes and control apoptosis, and as such are essential for multiplication of cancer cells. The mitochondrial oxidative phosphorylation system has a major effect on tumor progression [22, 24]. In addition, enhanced progression to malignancy was observed in cells with compromised mitochondrial integrity $[22,24]$. mtDNA mutations are significantly associated with the development of various types of cancer (for a review see [22]).

Clonal expansion of mutant mtDNA species was reported in $27-80 \%$ (average $54 \%$ ) of malignant tumor samples (for a review see [25]). In concert with this observation, we found that consensus sequences of mtDNA differ between tumor and blood from $\sim 58 \%$ of patients. Both particle-associated and free mtDNA are present in blood [26], potentially providing a convenient and minimally invasive way for the detection of cancer-related mitochondrial mutants [7]. As many cancer types, HCC is associated with clonally expanding mtDNA mutations [27-33]. The clonal expansion should affect genetic composition of mtDNA variants in 


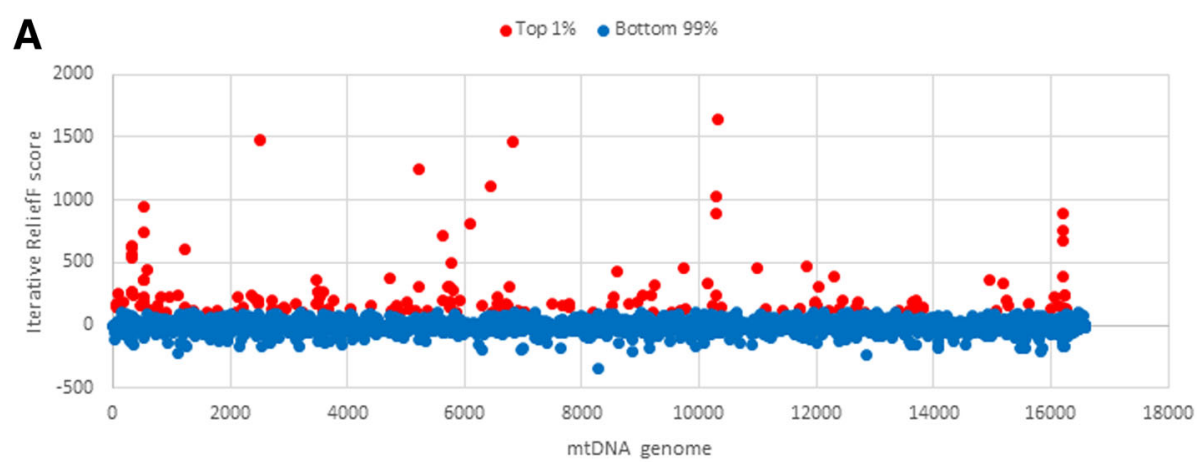

B

C
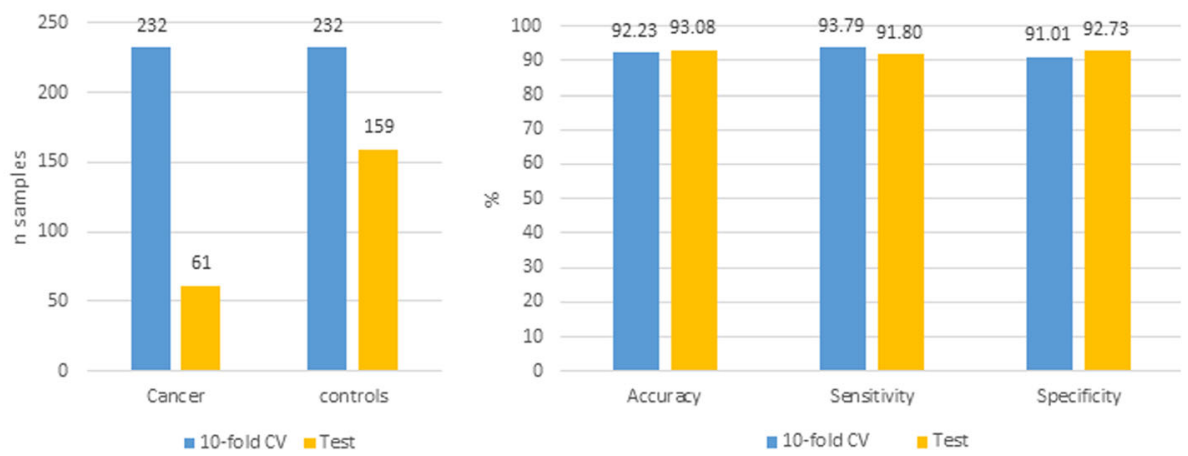

Fig. 6 Machine learning results. a Importance of each nucleotide position entropy in separating cancer and control samples. Only the sites within the top $1 \%$ scores (in red) were used for machine learning; b Distribution of samples; c Accuracy of the Random Forest classifier

blood. However, such an effect is not straightforward because mtDNA in blood has a very complex origin [26]. Moreover, requirements for efficient energy supply to rapidly replicating malignant cells constrains genetic composition of mitochondria in tumors [34].

The clonal expansion and genetic constraints coupled with a small size (16,569 bp) make mtDNA especially suitable for the accurate assessment of association of intra-host genetic heterogeneity, rather than specific mutations, with cancer. Application of heterogeneity profiles implemented here to the HCC detection overcomes the often-idiosyncratic presentation of specific mutations in cancer. Indeed, most tumor-specific variants (99.4\%) found in this study were present in less than $5 \%$ of HCC patient, thus impeding their use as general cancer markers. Complex and variable genetic nature of cancer is well established. It hinders the identification of specific mutations suitable for cancer diagnostics [3, 35, 36]. However, measures of intra-host genetic diversity in place of specific states of nucleotide sites mitigate the contribution of host-specific genetics to the detection of associations with cancer.

Tumor-specific mutations were present at low frequency in the blood of only $7.03 \%$ of patients. This finding indicates that the direct contribution of the tumor to the genetic composition of mtDNA in blood is limited, thus potentially confounding the detection of tumor-specific genetic variants in blood for cancer diagnostics. This concern becomes especially relevant when one considers a significant drop in mtDNA load in blood observed in this study and also reported elsewhere [37]. Nevertheless, the RF-classifiers generated here separated $\mathrm{HCC}$ and NC patients with accuracy exceeding 93\%, indicating the existence of a strong $\mathrm{HCC}$-specific genetic signal in intra-host mtDNA populations.

Genetic factors used in the RF-classifiers are fundamentally different from tumor-specific mutations identified from consensus mtDNA sequences. Only 11 tumor-specific sites were among the top 166 sites selected by entropy as relevant to the $\mathrm{HCC} / \mathrm{NC}$ classification, despite the fact that both sets of sites scattered along the entire mtDNA. Site entropy or its Z-score do not have information on a specific nucleotide state of a site, rather both measure nucleotide diversity at each site, thus reducing strong effects of specific mutations on associations captured by our models. There are many genetically diverse lineages of mtDNA. Although the $\mathrm{HCC}$ and NC datasets were matched by geographic location and mtDNA lineages, genetic differences among different genetic types of mtDNA may impede the identification of cancer-specific mutations, especially in a limited dataset. Entropy, however, represents a more general genetic information that can adequately trim genetic differences among mtDNA lineages, focusing 
nucleotide heterogeneity analyses on the identification of other than lineage-specific traits. Models generated using Z-scores performed as well as the entropy-based models. However, contribution of standardization achieved by application of Z-score to accuracy of models may become more apparent on more heterogeneous datasets.

Here, we applied machine-learning algorithms to extract genetic information from mtDNA for discriminating between HCC and NC. Application of the algorithms is routine in industrial and technological applications and only recently became successfully explored in clinical field [38, 39]. Machine learning presents a new opportunity to cancer diagnostics by shortcutting research from learning molecular mechanisms before developing applications to direct identification of reliable markers, thus accelerating development of accurate cancer detection.

We showed that tumor-specific mutant mtDNA species may be present at a very low concentration in blood. The detection of such minority variants can be achieved by UDS. Indeed, UDS has been applied to the efficient detection of tumor DNA [8] and to the detection of minority cancer-specific DNA variants [9]. However, a significant depletion of mtDNA has been reported for several cancer types such as bladder, breast, kidney, and liver cancer [37] making the detection of minority tumor-specific variants especially challenging. In agreement, we observed a $\sim 2$-fold decline in the number of reads mapped to mtDNA from tumor as compared to normal liver tissue, which further emphasizes potential difficulties in identification of specific mutant variants in tested blood. These observations indicate that consistent detection of minority variants is strongly contingent to a very high depth of sequencing. However, in difference to the detection of specific mutations, accurate estimation of site heterogeneity can be done at a moderate sequencing depth, thus providing a more reliable source of cancer-specific markers.

Uniform and adequate read coverage of the entire mtDNA can be challenging for the shotgun-based UDS. Sequencing of a single amplicon offers a greater control over the read coverage. However, it limits the mtDNA presentation to a single genomic region. Taking these observations in consideration, we hypothesized that such highly heterogeneous region of mtDNA as HVS1, may have sufficient genetic information to identify association with HCC. Indeed, the model constructed using HVS1 alone identified HCC versus NC with $83.22 \%$ $10 \mathrm{xCV}$ accuracy, indicating its applicability to the detection of HCC.

Finally, the observations presented here indicate significant differentiation of mtDNA heterogeneity between HCC and NC patients. Although showing separation between these 2 groups of patients, the data, however, do not allow to ascertain the strict HCC specificity of the classifiers. Detection of specific types of cancer versus general malignancy warrants additional investigation.

\section{Conclusions}

Sites contributing most to the association with HCC are scattered along the mtDNA genome, affecting all mitochondrial genes. The findings suggest that application of heterogeneity profiles of intra-host mtDNA variants from blood overcomes the complex association of specific mutations with cancer for the development of accurate, rapid, inexpensive and minimally invasive diagnostic detection of cancer.

The findings in this study suggest that genetic diversity of intra-host mtDNA in blood may serve as a generalizable marker for the accurate, rapid, inexpensive and minimally invasive diagnostic detection of cancer.

\section{Acknowledgements}

The authors are grateful to Scott Sammons (AMD, CDC), Bin Hu (AMD, CDC) and Victor Bolet (ITSO Application Hosting Branch, CDC) for their support and guidance on the development of computational tools accessible to the Public Health community.

\section{Funding}

All work and publication costs were funded by the Centers for Disease Control and Prevention, including the Division of Viral Hepatitis (DVH).

\section{Availability of data and materials} Not applicable.

\section{About this supplement}

This article has been published as part of BMC Medical Genomics Volume 12 Supplement 4, 2019: Selected articles from the 7th IEEE International Conference on Computational Advances in Bio and Medical Sciences (ICCABS 2017): medical genomics. The full contents of the supplement are available online at https://bmcmedgenomics.biomedcentral.com/articles/supplements/volume12-supplement-4.

\section{Authors' contributions}

DSC designed the study. VN, GS and DSC assembled the pipeline of bioinformatics tools for processing NGS data. DSC applied the pipeline and performed machine learning. DSC and YK analysed results and wrote the manuscript. All authors evaluated the final draft. All authors read and approved the final manuscript.

\section{Authors' information}

The findings and conclusions in this report are those of the authors and do not necessarily represent the official position of the Centers for Disease Control and Prevention.

\section{Ethics approval and consent to participate}

Informed consent was obtained for all participants (TCGA Research Network project \#9811).

\section{Consent for publication}

Not applicable.

Competing interests

The authors declare that they have no competing interests.

\section{Publisher's Note}

Springer Nature remains neutral with regard to jurisdictional claims in published maps and institutional affiliations. 


\section{Author details}

'Division of Viral Hepatitis, Centers for Disease Control and Prevention, Atlanta, GA, USA. ${ }^{2}$ Office of Advanced Molecular Detection, Centers for Disease Control and Prevention, Atlanta, GA, USA. ${ }^{3}$ CSRA, Inc, Corporate Blvd $\mathrm{NE}$, Atlanta, GA, USA.

Published: 6 June 2019

\section{References}

1. Cancer. http://www.who.int/mediacentre/factsheets/fs297/en/. Accessed 01 Feb 2018.

2. Larrea E, Sole C, Manterola L, Goicoechea I, Armesto M, Arestin M, Caffarel MM, Araujo AM, Araiz M, Fernandez-Mercado M, et al. New concepts in cancer biomarkers: circulating miRNAs in liquid biopsies. Int J Mol Sci. 2016; 17(5):627.

3. Yong E. Cancer biomarkers: written in blood. Nature. 2014;511(7511):524-6.

4. Leon SA, Shapiro B, Sklaroff DM, Yaros MJ. Free DNA in the serum of cancer patients and the effect of therapy. Cancer Res. 1977;37(3):646-50.

5. Vasioukhin V, Anker P, Maurice P, Lyautey J, Lederrey C, Stroun M. Point mutations of the $\mathrm{N}$-ras gene in the blood plasma DNA of patients with myelodysplastic syndrome or acute myelogenous leukaemia. Br J Haematol. 1994:86(4):774-9.

6. Sorenson GD, Pribish DM, Valone FH, Memoli VA, Bzik DJ, Yao SL. Soluble normal and mutated DNA sequences from single-copy genes in human blood. Cancer Epidemiol Biomark Prev. 1994;3(1):67-71.

7. Fliss MS, Usadel H, Caballero OL, Wu L, Buta MR, Eleff SM, Jen J, Sidransky D. Facile detection of mitochondrial DNA mutations in tumors and bodily fluids. Science. 2000;287(5460):2017-9.

8. Janku F, Zhang S, Waters J, Liu L, Huang HJ, Subbiah V, Hong DS, Karp DD, Fu S, Cai X, et al. Development and validation of an Ultradeep nextgeneration sequencing assay for testing of plasma cell-free DNA from patients with advanced cancer. Clin Cancer Res. 2017;23(18):5648-56.

9. Diehl F, Schmidt K, Choti MA, Romans K, Goodman S, Li M, Thornton K, Agrawal N, Sokoll L, Szabo SA, et al. Circulating mutant DNA to assess tumor dynamics. Nat Med. 2008;14(9):985-90.

10. The Cancer Genome Atlas. http://cancergenome.nih.gov/. Accessed 01 Mar 2016.

11. Genomes Project C, Auton A, Brooks LD, Durbin RM, Garrison EP, Kang HM, Korbel JO, Marchini JL, McCarthy S, McVean GA, et al. A global reference for human genetic variation. Nature. 2015;526(7571):68-74.

12. Andrews RM, Kubacka I, Chinnery PF, Lightowlers RN, Turnbull DM, Howell $\mathrm{N}$. Reanalysis and revision of the Cambridge reference sequence for human mitochondrial DNA. Nat Genet. 1999;23(2):147.

13. Calabrese C, Simone D, Diroma MA, Santorsola M, Gutta C, Gasparre G, Picardi E, Pesole G, Attimonelli M. MToolBox: a highly automated pipeline for heteroplasmy annotation and prioritization analysis of human mitochondrial variants in high-throughput sequencing. Bioinformatics. 2014; 30(21):3115-7.

14. Picardi E, Pesole G. Mitochondrial genomes gleaned from human wholeexome sequencing. Nat Methods. 2012;9(6):523-4.

15. Lo CC, Chain PS. Rapid evaluation and quality control of next generation sequencing data with FaQCs. BMC Bioinformatics. 2014;15:366.

16. Morelli MJ, Wright CF, Knowles NJ, Juleff N, Paton DJ, King DP, Haydon DT. Evolution of foot-and-mouth disease virus intra-sample sequence diversity during serial transmission in bovine hosts. Vet Res. 2013;44:12.

17. Shannon C. A mathematical theory of communication. Bell Syst Tech J. 1948;27:379-423

18. Robnik-Sikonja M, Kononenko I. Theoretical and empirical analysis of ReliefF and RReliefF. Mach Learn J. 2003;53:23-69.

19. Sun Y. Iterative RELIEF for feature weighting: algorithms, theories, and applications. IEEE Trans Pattern Anal Mach Intell. 2007;29(6):1035-51.

20. Breiman L. Random Forests. Mach Learn. 2001;45(1):5-32.

21. Pedregosa F, Varoquaux G, Gramfort A, Michel V, Thirion B, Grisel O, Blondel M, Prettenhofer P, Weiss R, Dubourg V, et al. Scikit-learn: machine learning in Python. J Mach Learn Res. 2011;12(Oct):2825-30.

22. van Gisbergen MW, Voets AM, Starmans MH, de Coo IF, Yadak R, Hoffmann RF, Boutros PC, Smeets HJ, Dubois L, Lambin P. How do changes in the $m t D N A$ and mitochondrial dysfunction influence cancer and cancer therapy? Challenges, opportunities and models. Mutat Res Rev Mutat Res. 2015;764:16-30.
23. Wallace DC. Mitochondrial DNA variation in human radiation and disease. Cell. 2015;163(1):33-8.

24. Wallace DC. Mitochondria and cancer. Nat Rev Cancer. 2012;12(10):685-98.

25. Khaidakov M, Shmookler Reis RJ. Possibility of selection against mtDNA mutations in tumors. Mol Cancer. 2005;4:36.

26. Chiu RW, Chan LY, Lam NY, Tsui NB, Ng EK, Rainer TH, Lo YM. Quantitative analysis of circulating mitochondrial DNA in plasma. Clin Chem. 2003;49(5): 719-26.

27. Wong $L$, Tan DJ, Bai RK, Yeh KT, Chang J. Molecular alterations in mitochondrial DNA of hepatocellular carcinomas: is there a correlation with clinicopathological profile? J Med Genet. 2004:41(5):e65.

28. Nishikawa M, Nishiguchi S, Shiomi S, Tamori A, Koh N, Takeda T, Kubo S, Hirohashi K, Kinoshita H, Sato E, et al. Somatic mutation of mitochondrial DNA in cancerous and noncancerous liver tissue in individuals with hepatocellular carcinoma. Cancer Res. 2001;61(5):1843-5.

29. Tamori A, Nishiguchi S, Nishikawa M, Kubo S, Koh N, Hirohashi K, Shiomi S, Inoue M. Correlation between clinical characteristics and mitochondrial Dloop DNA mutations in hepatocellular carcinoma. J Gastroenterol. 2004; 39(11):1063-8.

30. Wheelhouse NM, Lai PB, Wigmore SJ, Ross JA, Harrison DJ. Mitochondrial Dloop mutations and deletion profiles of cancerous and noncancerous liver tissue in hepatitis B virus-infected liver. Br J Cancer. 2005;92(7):1268-72.

31. Nomoto S, Yamashita K, Koshikawa K, Nakao A, Sidransky D. Mitochondrial D-loop mutations as clonal markers in multicentric hepatocellular carcinoma and plasma. Clin Cancer Res. 2002;8(2):481-7.

32. Zhang R, Zhang F, Wang C, Wang S, Shiao YH, Guo Z. Identification of sequence polymorphism in the D-loop region of mitochondrial DNA as a risk factor for hepatocellular carcinoma with distinct etiology. J Exp Clin Cancer Res. 2010;29:130.

33. Shawky R, Abdel-Gaffar T, Eladawy M, El-Etriby M, ElMoneiri M, Elhefnawy N, Elsherif R, Nour El-Din S. Mitochondrial alterations in children with chronic liver disease. Egypt J Med Hum Genet. 2010;1(2):143-51.

34. Ju YS, Alexandrov LB, Gerstung M, Martincorena I, Nik-Zainal S, Ramakrishna M, Davies HR, Papaemmanuil E, Gundem G, Shlien A, et al. Origins and functional consequences of somatic mitochondrial DNA mutations in human cancer. Elife. 2014;3:e02935.

35. Alexandrov LB, Nik-Zainal S, Wedge DC, Aparicio SA, Behjati S, Biankin AV, Bignell GR, Bolli N, Borg A, Borresen-Dale AL, et al. Signatures of mutational processes in human cancer. Nature. 2013:500(7463):415-21.

36. Alexandrov LB, Nik-Zainal S, Wedge DC, Campbell PJ, Stratton MR. Deciphering signatures of mutational processes operative in human cancer. Cell Rep. 2013;3(1):246-59,

37. Reznik E, Miller ML, Senbabaoglu Y, Riaz N, Sarungbam J, Tickoo SK, AlAhmadie HA, Lee W, Seshan VE, Hakimi AA, et al. Mitochondrial DNA copy number variation across human cancers. Elife. 2016;5:e10769.

38. Kononenko I. Machine learning for medical diagnosis: history, state of the art and perspective. Artif Intell Med. 2001;23(1):89-109.

39. Foster KR, Koprowski R, Skufca JD. Machine learning, medical diagnosis, and biomedical engineering research - commentary. Biomed Eng Online. 2014; 13:94.

Ready to submit your research? Choose BMC and benefit from:

- fast, convenient online submission

- thorough peer review by experienced researchers in your field

- rapid publication on acceptance

- support for research data, including large and complex data types

- gold Open Access which fosters wider collaboration and increased citations

- maximum visibility for your research: over $100 \mathrm{M}$ website views per year

At $\mathrm{BMC}$, research is always in progress.

Learn more biomedcentral.com/submission 